\title{
Música y territorio en la etnomusicología latinoamericana: III Simposio del Comité Nacional Chileno del Consejo Internacional para la Música Tradicional (ICTM-Chile)
}

\author{
por \\ Ignacio Soto-Silva \\ Departamento de Humanidades y Artes, Universidad de Los Lagos, Chile \\ Vicepresidente ICTM-Chile \\ ignacio.soto@ulagos.cl \\ Leonardo Díaz-Collao \\ Departamento de Humanidades y Artes, Universidad de Los Lagos, Chile \\ Secretario ICTM-Chile \\ leonardo.diaz@ulagos.cl \\ Javier Silva-Zurita \\ Departamento de Humanidades y Artes, Universidad de Los Lagos, Chile \\ Presidente ICTM-Chile \\ javier.silva@ulagos.cl
}

Durante los días 27, 28 y 29 de octubre de 2021 se realizó el III Simposio ICTM-Chile 2021 "Etnomusicología y Territorios", organizado de manera conjunta por el Comité Nacional Chileno del Consejo Internacional para la Música Tradicional (ICTM-Chile) y la Academia de Artes Musicales de la Universidad Católica de Temuco, particularmente por su Diplomado de Estudios en Música, Cultura y Sociedad. Por segundo año consecutivo, y debido a las condiciones sanitarias derivadas de la pandemia COVID-19, el encuentro fue realizado de manera telemática, siendo transmitido por el canal de YouTube de la Academia de Artes Musicales de la Universidad Católica de Temuco.

Esta actividad buscó reunir a investigadores/as, docentes, cultores/as y gestores/as, entre otros actores, con el objeto de discutir los enfoques, metodologías y modos de abordar y comprender los lugares y espacios donde las prácticas musicales ocurren. El concepto de territorio ha experimentado grandes cambios en las últimas décadas, pasando desde una concepción que lo limitaba a aspectos geográficos medianamente estáticos, hacia una visión que lo expande al incorporar su dimensión social y naturaleza dinámica. En la era de la globalización, el concepto de territorio está en constante construcción; ya no solo abarca los espacios y lugares físicos donde se desenvuelven los músicos y sus audiencias, sino también las construcciones y proyecciones acerca de un lugar natural, imaginario o virtual que se vincula a una cultura o grupo de personas.

Quisiéramos destacar la diversidad de propuestas recibidas para este simposio, las que abarcaron temáticas variadas. Se logró reunir a investigadores de distintitos países, los que trataron problemáticas 
de la investigación musical vinculadas a América Latina y sus constructos territoriales. Esto nos llevó a organizar las presentaciones en seis bloques distribuidos a lo largo de tres días, cuestión que consideramos positiva en tanto muestra un creciente interés por las problemáticas vinculadas a la disciplina. Las jornadas se organizaron de la siguiente forma:

Miércoles 27 de octubre - Mañana ${ }^{1}$

9:00-9:10 Palabras de bienvenida. Javier Silva-Zurita, Presidente ICTM-Chile, y Fernando Sandoval, Director Academia de Artes Musicales, Universidad Católica de Temuco.

Tradición, música y migración / Moderadora: Lucero Burón, Universidad Católica de Temuco 9:10-9:30 "Con garra de tigre: migración y endemismo". María Fernanda del Peón Pacheco, Universidade de Aveiro/ INET-md.

9:30-9:50 "La música de gaita en Colombia: territorios ausentes". Urián Sarmiento, Conservatorio Universidad Nacional de Colombia.

9:50-10:10 "La gaita colombiana: migración y actos de nostalgia”. Katerine Zamora, Centro de Investigación Musical del Conservatorio Adolfo Mejía-CIMCAM.

10:10-10:30 Preguntas y discusión.

Territorios, diáspora y música / Moderadora: Andrea Chamorro, Universidad de Tarapacá

10:30-10:50 "Propuesta metodológica para el estudio de la diáspora croata en Chile: aproximación inicial al desarrollo del folclor como elemento central en la construcción de una identidad migrante”. Jan Koplow y Tamara Bulicic, Pontificia Universidad Católica de Chile.

10:50-11:10 "De la cabeza a la cola y de vuelta: la danza del león chino como performance de chineseness en Santiago, Chile”. Tamara Bulicic, Pontificia Universidad Católica de Chile.

11:10-11:30 "Si acá también somos andinos: Territorio transnacional andino y la danza del caporal”. Javiera Benavente, Pontificia Universidad Católica de Chile.

11:30-11:50 Preguntas y discusión.

Miércoles 27 de octubre - Tarde ${ }^{2}$

17:00-18:20 Mesa redonda: "Sonidos de una presencia negada: territorialidades del tumbe afroariqueño”. Mariana León Villagra, Investigadora independiente, Colectivo BosquesEnSilencio; Ignacio Carrasco Meza, Universidad de Tarapacá, Comparsa afroariqueña "Tumba Carnaval"; Daniel Domingo Gómez, Universidad de Santiago de Chile; y Ricardo Amigo Dürre, Universidad de Chile.

18:20-19:20 Lanzamiento del libro Wechemapu. Autobiografía de un imaginario. Iris Rumián, Ponciano Rumián (autores) e Ignacio Soto-Silva (moderador, Universidad de Los Lagos)

1 Video disponible en: https://youtu.be/v4t4sM97axo [acceso: 26 de noviembre de 2021].

2 Video disponible en: https://youtu.be/_JUeMnwL8jA [acceso: 26 de noviembre de 2021]. 


\section{Jueves 28 de octubre - Mañana ${ }^{3}$}

\section{Sonido y memoria / Moderador: José Pérez de Arce, Universidad de Chile}

9:00-9:20 "Arqueomusicología en el Cachapoal: Hacia una etnología del sonido a través de la reconstrucción de aerófonos líticos en el Chile central”. Diep Sarrúa Yurazeck, Centro de Investigación y Creación Musical Carlos Isamitt.

9:20-9:40 "Don Reinaldo Lagos y sus registros sonoros etnográficos en el Alto Loa y Atacama Grande, Región de Antofagasta-Chile”. Gustavo Araya, GEMAndina, La Serena-Chile.

9:40-10:00 "Metodología Bancos de Memoria". Lucía Ibáñez y Urián Sarmiento, Corporación Cultural Sonidos Enraizados, Colombia.

10:00-10:20 Preguntas y discusión.

Música, identidad y territorio / Moderadora: Katherine Zamora, CIMCAM

10:40-11:00 "Construcción de identidades champetúas en Champetú y Champeta en Pasta". Nathaly Gómez, Pontificia Universidad Javeriana, Colombia.

11:00-11:20 Preguntas y discusión.

$$
\text { Jueves } 28 \text { de octubre - Tarde } 4
$$

\section{Moderador: Pablo Rojas Sahurie, Universidad de Viena}

17:00-17:20 "Yahora, ¿pa' dónde nos vamos?: Repensando el campo etnográfico a través de TikTok". Juan Bermúdez, Universidad de Viena.

17:20-17:40 "Música, cultura y medioambiente: Algunas aproximaciones teóricas y metodológicas en ecomusicología". Mauricio Valdebenito, Universidad de Chile.

17:40-18:00 "Toki Rapa Nui: Tallando una nueva identidad territorial a través de la educación musical". Moira Fortin, Universidad de Otago.

18:00-18:20 Preguntas y discusión.

Viernes 29 de octubre - Mañana ${ }^{5}$

Política, música y descolonización / Moderadora: Daniela Fugellie, Universidad Alberto Hurtado 9:00-9:30 "Vocalidad como estrategia de descolonización sonora del repertorio coral: el caso del Osún Réquiem de Calixto Álvarez". Marco Hugo Marchant, Pontificia Universidad Católica de Chile.

9:30-10:00 "Ópera chilena, cultura originaria y territorio. Descentralización y democratización del género lírico desde la reivindicación de los pueblos indígenas y la participación comunitaria”. Constanza Arraño, Universidad Alberto Hurtado.

10:00-10:20 Preguntas y discusión.

3 Video disponible en: https://youtu.be/I4yynAjtows [acceso: 26 de noviembre de 2021].

4 Video disponible en: https://youtu.be/zhIfrAcfFZg [acceso: 26 de noviembre de 2021].

5 Video disponible en: https://youtu.be/o9gXnL1hplU [acceso: 26 de noviembre de 2021]. 


\section{Música popular, música mapuche y territorio / Moderador: Jacob Rekedal,} Universidad Alberto Hurtado

10:20-10:40 "La generación de folkloristas de mediados del siglo XX en La Araucanía profunda: Identidad y música popular de las chilenas y chilenos sin voz". Mathias Órdenes y Mario Samaniego, Núcleo de Investigación en Estudios Interculturales e Interétnicos (NEII), Universidad Católica de Temuco.

10:40-11:00 "Juan de Dios Curilem Millaguir: Primer musicólogo mapuche”. Laura Jordán, Pontificia Universidad Católica de Valparaíso, y Andrea Salazar, Universidad de Chile.

11:00-11:20 "Los ritmos de la interferencia: identidades culturales, descolonización y rap mapuche". Alejandro Rossi, University of California, Davis.

11:20-11:40 Preguntas y discusión.

Viernes 29 de octubre - Tarde 6

\section{Moderador: Leonardo Díaz-Collao, Diplomado de Estudios en Música, Cultura y Sociedad, Universidad Católica de Temuco}

17:00-17:20 "La escucha como territorio: performatividad sonora y artivismos transfeministas en Latinoamérica". Victoria Polti, Universidad de Buenos Aires.

17:20-17:40 "Fuego, desmonumentalización y topofilia: El caso de la Banda Dignidad (2019-2021)". Christian Spencer, Centro de Investigación en Artes y Humanidades, Universidad Mayor.

17:40-18:00 Preguntas y discusión.

18:00-19:00 Conferencia: "Experiencia aural urbana y territorios sonoros: encuentros, resonancias y subjetividades al habitar la ciudad". Natalia Bieletto-Bueno, Centro de Investigación en Artes y Humanidades, Universidad Mayor.

La actividad contó con siete mesas temáticas, una mesa redonda, una presentación de libro y una conferencia de cierre. El miércoles 27 de octubre se inició con la mesa titulada Tradición, música y migración. En dicha instancia se abordaron tres experiencias relativas a las prácticas musicales en el contexto de la migración, con trabajos que apuntaron principalmente al rol de la gaita colombiana en aquellos procesos y cómo la presencia de este instrumento genera espacios de nostalgia en los cultores. Posteriormente, la mesa titulada Territorios, diáspora y música abordó cuestiones como las construcciones identitarias migrantes asociadas al folclore croata, a la danza del león chino en la ciudad de Santiago y a las problemáticas vinculadas al territorio transnacional andino.

Durante la jornada de la tarde se llevó a cabo la mesa redonda titulada Sonidos de una presencia negada: territorialidades del tumbe afroariqueño, la que abordó distintas aproximaciones al estudio de la música de las comunidades afrodescendientes en el norte de Chile. La jornada finalizó con la presentación del libro Wechemapu: autobiografía de un imaginario, la que estuvo a cargo de sus autores Iris Rumian y Ponciano Rumian. Es interesante destacar que este libro responde a un proceso de reflexión de la agrupación en sus cuarenta y cinco años de trayectoria, cuyo texto da cuenta de un relato desde los cultores que nos invita a repensar nuestras etnografías.

El jueves 28 de octubre por la mañana se desarrollaron las mesas temáticas Sonido y memoriay Música, identidad y territorio. En la primera se compartieron experiencias de reproducción de instrumentos musicales prehispánicos de la zona del Cachapoal, de construcción participativa de repositorios sonoros con indígenas y afrocolombianos, y acerca de los antecedentes de los registros etnográficos realizados por Reinaldo Lagos Carrizo. En la segunda mesa se presentaron reflexiones en torno a la construcción de identidades champetúas en Cartagena, Colombia. Por la tarde se realizaron tres ponencias que abordaron experiencias de enseñanza musical en Rapa Nui, una revisión crítica de ecomusicología y una reflexión acerca de trabajo etnográfico y plataformas digitales.

6 Video disponible en: https://youtu.be/ZNMD_vgHw3I [acceso: 26 de noviembre de 2021]. 
El viernes 29 de octubre se realizaron las últimas sesiones del simposio. La primera mesa del día se tituló Política, música y descolonización, en donde se discutieron tópicos relacionados con el rol de la vocalidad en procesos de descolonización, así como la reivindicación de los pueblos indígenas por medio de la democratización del género lírico. En la segunda mesa de la mañana, Música popular, música mapuche y territorio, se discutió acerca de las interacciones entre el espacio y la música popular. Las ponencias trataron cuestiones como el folclore en La Araucanía profunda durante el siglo xx, los antecedentes históricos de Juan de Dios Curilem Millaguir, quien podría ser considerado el primer musicólogo mapuche, y ciertos procesos de descolonización y reafirmación identitaria observados en el rap mapuche.

La jornada final de este encuentro se configuró a partir de tres ponencias acerca del rol del territorio en la ciudad y sus vínculos con procesos de reivindicaciones sociales, tales como el estallido social en Chile y el artivismo transfeminista en América Latina. La actividad culminó con la conferencia de Natalia Bieletto-Bueno, titulada "Experiencia aural urbana y territorios sonoros: encuentros, resonancias y subjetividades al habitar la ciudad”. En la charla, la musicóloga reflexionó en torno al rol del sonido, la escucha, el cuerpo y la afectividad en la construcción del lugar y de subjetividades en espacios urbanos.

Quisiéramos destacar el crecimiento que han experimentado los encuentros realizados por ICTMChile desde su creación en el 2019. En un ejercicio de sistematización que hemos procurado realizar durante las distintas sesiones de este encuentro (Soto-Silva et al., 2020; Soto-Silva et al., 2021), hemos podido evidenciar cómo esta instancia de diálogo entre investigadores, cultores, gestores y educadores ha adquirido un creciente interés dentro de la comunidad sudamericana. Nos complace poder abrir espacios de discusión académica, así como también esperamos poder continuar con la descentralización de estas instancias. No es casual que nuestro primer encuentro se haya realizado en Valparaíso, el segundo en la Universidad de Los Lagos y el aquí reseñado en la Universidad Católica de Temuco. En la medida que logremos descentralizar la investigación musical y los polos de desarrollo de esta, fortaleceremos la discusión disciplinar y las miradas que la componen.

Como queda patente en el título del simposio, propusimos reflexionar respecto de dos conceptos principales: territorio y etnomusicología. El interés por discutir la categoría "territorio" nació de su importante presencia no solo en discusiones académicas de diversas áreas, sino también en el discurso público. Como ocurre con otros conceptos, "territorio" se utiliza en ocasiones como un término genérico y no se precisan definiciones disciplinares u operativas. Nos pareció que invitar a debatir acerca de diversos temas desde este pie forzado ayudaría a clarificar las múltiples nociones del concepto con las que trabajamos. Sin embargo, las diversas aproximaciones teóricas que se desplegaron durante el simposio invitan a complejizar la categoría, así como otros términos emparentados como lugar, espacio, entre otros, y a especificar su relación con la música, el sonido y la escucha. El segundo concepto clave que nos hemos esmerado en posicionar por medio de los simposios, "etnomusicología", proponemos comprenderlo siempre de un modo dialogante y con límites -en buena hora- difusos. Nos parece que defender a la etnomusicología como si se tratase de una disciplina bien definida en lo teórico y metodológico es extemporáneo e infructuoso. Como demuestra la variedad de enfoques y disciplinas en las que se reflexionó durante los días del encuentro sobre territorio, música y sonido, reconocemos que la etnomusicología es uno de nuestros lugares de enunciación, pero que debe aspirar a una práctica inter, trans o, bien, posdisciplinaria.

\section{BIBLIOGRAFÍA}

Soto-Silva, Ignacio, Jacob Rekedal, Javier Silva-Zurita y Leonardo Díaz-Collao

2021 Reflexiones sobre etnomusicología y enseñanza musical en Chile: segundo simposio del Comité Nacional Chileno del Consejo Internacional para la Música Tradicional (ICTMChile). Revista Musical Chilena LXXV/235, pp. 257-260.

Soto Silva, Ignacio, Jacob Rekedal y Javier Silva-Zurita

2020 Diálogos y reflexiones sobre la etnomusicología en Chile: reunión fundacional del Comité Chileno del Consejo Internacional para la Música Tradicional (ICTM-Chile). Revista Musical Chilena, LXXIV/233, pp. 196-200. 